\title{
General Periodic Boundary Value Problem for Systems
}

\author{
Mohammed Elnagi ${ }^{1,2}$ \\ ${ }^{1}$ Department of Mathematics, Faulty of Educations, Khartoum University, Omdurman, Sudan \\ ${ }^{2}$ Department of Mathematics, Northwest Normal University, Lanzhou, China \\ Email: mohdnajy@hotmail.com
}

Received May 20, 2012; revised July 2, 2012; accepted July 9, 2012

\section{ABSTRACT}

The paper deals with the existence of nonzero periodic solution of systems $\begin{cases}u^{\prime \prime}+k^{2} u=f(t, u), & t \in(0, T), T>0, \\ u(0)=\alpha u(T), & u^{\prime}(0)=\beta u^{\prime}(T),\end{cases}$ where $k \in\left(0, \frac{\pi}{T}\right), \alpha, \beta$ are $n \times n$ real nonsingular matrices, $u=\left(u_{1}, \cdots, u_{n}\right)$, $f(t, u)=\left(f_{1}(t, u), \cdots, f_{n}(t, u)\right) \in C\left([0, T] \times \mathbb{R}_{+}^{n}, \mathbb{R}_{+}\right)$is periodic of period $T$ in the $t$ variable are continuous and nonnegative functions. We determine the Green's function and prove that the existence of nonzero periodic positive solutions if one of $\lim _{\|u\| \rightarrow 0} \frac{f_{i}(t, u)}{\|u\|}>\lambda_{1}$. In addition, if all $\lim _{\|u\| \rightarrow 0} \frac{f_{i}(t, u)}{\|u\|}<\lambda_{1}, i=(1, \cdots, n)$ where $\lambda_{1}$ is the principle eigenvalues of the corresponding linear systems. The proof based on the fixed point index theorem in cones. Application of our result is given to such systems with specific nonlinearities.

Keywords: Systems; Principle Eigenvalues; Positive Solutions; Green's Function; Fixed Point Index Theorem in Cones

\section{Introduction}

In this paper, we study the existence of nonzero positive periodic solution of systems

$$
\begin{cases}u^{\prime \prime}+k^{2} u=f(t, u), & t \in(0, T), T>0 \\ u(0)=\alpha u(T), & u^{\prime}(0)=\beta u^{\prime}(T)\end{cases}
$$

where $k \in\left(0, \frac{\pi}{T}\right), \alpha, \beta$ are $n \times n$ real nonsingular matrices, $u=\left(u_{1}, \cdots, u_{n}\right)$, and

$$
f(t, u)=\left(f_{1}(t, u), \cdots, f_{n}(t, u)\right) \in C\left([0, T] \times \mathbb{R}_{+}^{n}, \mathbb{R}_{+}\right)
$$

are periodic of period $T$ in the $t$, are continuous and nonnegative functions and $\mathbb{R}_{+}=[0, \infty)$.

Beginning with the paper of Erbe and Palamides [1], obtained the sufficient conditions for existence solution of the systems of nonlinear boundary value problem

$$
\left\{\begin{array}{l}
u^{\prime \prime}=f\left(t, u, u^{\prime}\right), \quad t \in(0,1) \\
u(0)=Q_{0} u(1), \quad u^{\prime}(0)=Q_{1} u^{\prime}(1)
\end{array}\right.
$$

where $f:[0,1] \times E^{2 n} \rightarrow E^{n}$ is continuous ( $E^{n}$ is $n$-dimensional real Euclidean space) and $Q_{0}, Q_{1}$ are nonsingular matrices, with $Q_{0}$ orthogonal matrix, Erbe and
Palamides generalize earlier conditions of Bebernes and Schmitt [2] for periodic case. Erbe and Schmitt [3] extend the results in [1] established the sufficient conditions for existence solution of the systems (1.2). The results in $[1,3]$ were obtained via a modifications of a degree-theoretic approach and Leray-Schuader degree and eliminates the modified function approach respectively. None of these earlier results use Green's function and the first eigenvalues of the corresponding to the linear systems of (1.1).

There has been progress in the study of the existence of positive solutions of system problem. If $\alpha=\beta=I=$ identity, then $(1,1)$ reduces to the usual periodic boundary value problem for which the literature in both the scalar and systems versions is very extensive (We refer to [4-22] and references therein). For instance a recent paper, Wang [4] obtained the existence of periodic solution of a class of non-autonomous second-order systems

$$
u^{\prime \prime}+\mu u+f(t, u)=0, \quad t \in(0, T), T>0
$$

where

$$
\begin{aligned}
& u=\left(u_{1}, \cdots, u_{n}\right), \\
& f(t, u)=\left(f_{1}(t, u), \cdots, f_{n}(t, u)\right) \in C\left([0, T] \times \mathbb{R}^{n}, \mathbb{R}^{n}\right)
\end{aligned}
$$

are periodic of period $T$ in the $t$, and $\mu$ is a constant, 
if $\lim _{|u| \rightarrow 0} \frac{f_{i}(t, u)}{|u|}=0, i=(1, \cdots, n)$ and $f(t, u)$, is bounded below or above for appropriate ranges of $\mu$, via fixed point theorem in cones. Franco and Webb [5] established the existence of $T$-periodic solutions for systems of (1.1) with $\alpha=\beta=I=$ identity, in the boundary conditions, where $k \in \mathbb{R}$ and $f(t, u)$ is a continuous vector valued function, periodic in $t$ with period $T$, and $f$ is allowed to have a singularity when $u=0$. Non-singular systems, which are included in the same framework that we study here (i.e., they can be reduced to a Hammerstein integral system with positive kernel), have been considered using some other approaches based on fixed point theorems in conical shells, but previously it has always been assumed that the nonlinearity $f(t, u)$ has a constant sign behaviour: $f_{i}(t, u) \theta_{i} \geq 0$ where

$\theta_{i} \in\{1,-1\}$ for $0 \leq i \leq n$ (see [6]) with $\alpha=\beta=I=$ identity, in the boundary conditions. For systems problem see also [7-12] and references therein.

Even in the scalar case the existence of periodic solutions for problems with nonsingular and singular case has commanded much attention in recent years (see [13-22] and references therein. In particular, in [13-15] fixed point theorems in conical shells are used to obtain existence and multiplicity results, some of these are improved in this paper. In this notes, we prove result in the case where $f$ has no singularity. In scalar case problem see [16-22] and references therein.

Motivated by these problems mentioned above, we study the existence of nonzero positive solution of (1.1) while we assume that if one components satisfy

$\lim _{\mid u \rightarrow \rightarrow 0} \frac{f_{i_{0}}(t, u)}{\|u\|}>\lambda_{1}, i_{0} \in\{1, \cdots, n\}$, and all components of nonlinearity are $\lim _{|u| \rightarrow 0} \frac{f_{i}(t, u)}{\|u\|}<\lambda_{1}, \quad i=\{1, \cdots, n\}$, where $\lambda_{1}$, is the largest characteristic value of the linear system corresponding to (1.1). The approach is to use the theory of fixed point index for compact maps defined on cones [23]. To apply this theory one needs to find the Green's function. Our purpose here is prove that (1.1) has nontrivial nonnegative solution, assuming the following conditions:

$$
\begin{aligned}
& \text { (H1) } 0<k<\frac{\pi}{T}, \\
& \text { (H2) } \alpha, \beta \geq 0, \alpha \beta \geq 0, \alpha-\beta>0, \alpha+\beta>0
\end{aligned}
$$

and
$2 k(1+\alpha \beta-(\alpha+\beta) \cos k T)>0$,

(H3) $f(t, u)=\left(f_{1}(t, u), \cdots, f_{n}(t, u)\right) \in C\left([0, T] \times \mathbb{R}_{+}^{n}, \mathbb{R}_{+}\right)$

are continuous and periodic of period $T$ in the $t$ variable and $f_{i}(t, u) \neq 0, i=(1, \cdots, n)$ on any subinterval of $[0, T]$.

(H4) There exits $i_{0} \in\{1, \cdots, n\}$ such that

$$
\lim _{\|u\| \rightarrow 0} \frac{f_{i_{0}}(t, u)}{\|u\|}>\lambda_{1},
$$

where $u=\left(u_{1}, \cdots, u_{n}\right) \in \mathbb{R}_{+}^{n}$ and $\|u\|=\sum_{i=1}^{n} \sup \left|u_{i}\right|$, and $\lambda_{1}$ is the largest characteristic value of the linear system corresponding to (1.1),

(H5) For all $i \in(1, \cdots, n)$,

$$
\lim _{\|u\| \rightarrow 0} \frac{f_{i}(t, u)}{\|u\|}<\lambda_{1}
$$

where $u=\left(u_{1}, \cdots, u_{n}\right) \in \mathbb{R}_{+}^{n}$ and $\|u\|=\sum_{i=1}^{n} \sup \left|u_{i}\right|$, and $\lambda_{1}$ is the largest characteristic value of the linear system corresponding to (1.1).

Remark 1.1. The assumptions (H4) and (H5) appeared in Lan [24].

Remark 1.2. The nonzero positive solution has been studied by Lan [24] and Hai and Wang [25].

Throughout this paper, we will use the notation $\mathbb{R}_{+}=[0, \infty), \mathbb{R}_{+}^{n}=\prod_{i=1}^{n} \mathbb{R}_{+}$, and denote by $|u|=\sum_{i=1}^{n}\left|u_{i}\right|$ the usual norm of $\mathbb{R}_{+}^{n}$ for $u=\left(u_{1}, \cdots, u_{n}\right) \in \mathbb{R}_{+}^{n}$, $f(t, u)=\left(f_{1}(t, u), \cdots, f_{n}(t, u)\right)$ and $i=(1, \cdots, n)$.

\section{Preliminaries}

In this section, we shall introduce some basic lemmas which are used throughout this paper.

Lemma 2.1. Let $(H 1)$ and $(H 2)$ holds. Let $\Delta=2 k(1+\alpha \beta-(\alpha+\beta) \cos k T)$ then for $v_{i} \in C[0, T]$, the periodic boundary value problems problem

$$
\begin{cases}u_{i}^{\prime \prime}+k^{2} u_{i}=v_{i}, & t \in(0, T), T>0, \\ u_{i}(0)=\alpha u_{i}(T), & u_{i}^{\prime}(0)=\beta u_{i}^{\prime}(T),\end{cases}
$$

has a unique solution

$$
u_{i}=\int_{0}^{T} G_{i}(t, s) v_{i} \mathrm{~d} s,
$$

where

$$
G(t, S)=\left\{\begin{array}{l}
2 \alpha \beta \sin k(t-s)+(\alpha-\beta) \sin k(T-s-t)+(\alpha+\beta) \sin k(T+s-t), 0 \leq s \leq t \leq T, \\
2 \alpha \beta \sin k(s-t)+(\alpha-\beta) \sin k(T-s-t)+(\alpha+\beta) \sin k(T+t-s), 0 \leq t \leq s \leq T
\end{array}\right.
$$


where $i=(1, \cdots, n)$.

Proof. Consider the scalar periodic boundary value problems of (2.1) and let $u_{1}(t)$ and $u_{2}(t)$ be linearly distinct solutions of the scalar equation of (2.1) and consider the function

$$
\begin{aligned}
F(t, s) & =A u_{1}(t)+B u_{2}(t) \\
& \pm\left(\frac{u_{1}(t) u_{2}(s)-u_{1}(s) u_{2}(t)}{2\left(u_{1}(s) u_{2}^{\prime}(s)-u_{1}^{\prime}(s) u_{2}(s)\right)}\right)
\end{aligned}
$$

where the positive sign is taken when $0 \leq t \leq s$, and the negative sign when $s \leq t \leq T$ we can obtain this result by routine substitutions of scalar boundary conditions, we do not state it here.

Lemma 2.2. Let conditions ( $H 1)$ hold, then $G_{i}(t, s)$, $i=(1, \cdots, n)$, is continuous and positive on $[0, T] \times[0, T]$, and we can find it's positive minimum value $A_{i}$ and maximum value $B_{i}$ of $G_{i}(t, s), i=(1, \cdots, n)$ by

$$
0<A_{i}=\min _{t, s \in[0, T]} G_{i}(t, s), \quad B_{i}=\max _{t, s \in[0, T]} G_{i}(t, s),
$$

and

$$
0<\sigma_{i}=\frac{A_{i}}{B_{i}}, \quad i=(1, \cdots, n) .
$$

Proof. It is easy to check that $G_{i}(t, s)$, is continuous and positive on $[0, T] \times[0, T], \quad i=(1, \cdots, n)$. .

It is clear that the problem (1.1) has a solution $u$ if and only if $u$ solves the operator equation

$$
A u(t):=\int_{0}^{T} G_{i}(t, s) f_{i}(s, u(s)) \mathrm{d} s
$$

It is easy to verify that the operator $A$ is completely continuous.

We define $L: C\left([0, T] ; \mathbb{R}^{n}\right) \rightarrow C\left([0, T] ; \mathbb{R}^{n}\right)$ corresponding to linear equation of (1.1) by

$$
\begin{aligned}
& L u(t)=\int_{0}^{T} G_{1}(t, s) u_{1}(s) \mathrm{d} s, \cdots, \int_{0}^{T} G_{n}(t, s) u_{n}(s) \mathrm{d} s, \\
& t \in[0, T]
\end{aligned}
$$

where $u(t)=\left(u_{1}(t), \cdots, u_{n}(t)\right) \in \mathbb{R}^{n} \quad$ and $\quad G_{i}(t, s)$ is Green's function define in $\left(2.1^{*}\right)$, and define

$$
\left(\Gamma u_{1}\right)(t)=\int_{0}^{T} G_{1}(t, s) u_{1}(s) \mathrm{d} s
$$

and

$$
\left(\Gamma u_{n}\right)(t)=\int_{0}^{T} G_{n}(t, s) u_{n}(s) \mathrm{d} s,
$$

where $\Gamma: C([0, T]) \rightarrow C([0, T])$ is completely continuous.

Remark 2.1. Equations (2.2) appeared in [26].

It is known that $\Gamma^{*}: C([0, T]) \rightarrow C([0, T])$, is a bounded and surjective linear operator and has a unique extension, denoted by $\Gamma$, to $C([0, T])$. We write

$$
e=\Gamma^{*} v_{0}, v_{0}(t) \equiv 1, v \in C([0, T]) .
$$

It is known that $e$ is an interior point of the positive cone $P_{1}$ in $C([0, T])$, where

$$
P_{1}=\{u \in C([0, T]): u(t) \geq 0, t \in[0, T]\} .
$$

Lemma 2.3. [24] $\Gamma: C([0, T]) \rightarrow C([0, T])$ is a compact linear operator such that $\Gamma\left(P_{1}\right) \subset P_{1}$ and for each $v \in P_{1} \backslash\{0\}$ there exists $\alpha_{v}>0$ such that $\Gamma v \geq \alpha_{v} e$.

By Lemma 2.3 and the well-known Krein-Rutman theorem (see [23, Theorem 3.1] or [27], it is easy to see that $\mu_{1} \in(0, \infty)$ and there exists $\varphi_{1} \in P_{1} \backslash\{0\}$ such that

$$
\varphi_{1}=\mu_{1} \Gamma \varphi_{1}
$$

where $\mu_{1}=1 / r(\Gamma)$ and $r(\Gamma)$ is the spectral radius of $\Gamma$.

We use the following maximum norm in $\mathbb{R}^{n}$ :

$$
|u|=\max \left\{\left|u_{i}\right|: i \in(1, \cdots, n)\right\}
$$

where $u(t)=\left(u_{1}(t), \cdots, u_{n}(t)\right)$. We denote by $\left(C([0, T]) ; \mathbb{R}^{n}\right)$ the Banach space of continuous functions from $[0, T]$ into $\mathbb{R}^{n}$ with norm $\|u\|=\max \left\{\left\|u_{i}\right\|: i \in(1, \cdots, n)\right\}$, where $u(t)=\left(u_{1}(t), \ldots, u_{n}(t)\right)$ for $t \in[0, T]$.

We use the standard positive cone in $\left(C([0, T]) ; \mathbb{R}^{n}\right)$ defined by

$$
P=C([0, T]) ; \mathbb{R}^{n}
$$

We can write $L: C\left([0, T] ; \mathbb{R}^{n}\right) \rightarrow C\left([0, T] ; \mathbb{R}^{n}\right)$ defined in (2.2) as operator equations

$$
(L u)(t):=\left(\left(\Gamma u_{1}\right)(t), \cdots,\left(\Gamma u_{n}\right)(t)\right)
$$

where $\left(\Gamma u_{1}\right)(t)$ and $\left(\Gamma u_{n}\right)(t)$ are define above and define a Nemytskii operator

$$
(F u)(t):=\left(f_{1}(t, u(t)), \cdots, f_{n}(t, u(t))\right) .
$$

It is easy to verify that (1.1) is equivalent to the following fixed point equation:

$$
u(t)=(L F)(t):=A u(t), t \in[0, T]
$$

Note that (2.10) same as $(I)$.

Recall that a solution $u \in\left(C([0, T]) ; \mathbb{R}^{n}\right)$ of $(1.1)$ is said to be a nonzero positive solution if $u \in P \backslash\{0\}$; that is, $u \in\left(C([0, T]) ; \mathbb{R}^{n}\right)$ and $u(t)=\left(u_{1}(t), \cdots, u_{n}(t)\right)$ satisfies $u_{i}(t) \geq 0$ for $t \in[0, T]$ and $i=(1, \cdots, n)$ and there exists such that $k \in(1, \cdots, n) \quad u_{k} \neq 0$ on $[0, T]$.

Let $\rho>0$ and let 


$$
P_{\rho}=\{u \in P:\|u\|<\rho\}, \quad \bar{P}_{\rho}=\{u \in P:\|u\| \leq \rho\}
$$

and

$$
\partial P_{\rho}=\{u \in P:\|u\|=\rho\} .
$$

We need some results from the theory of the fixed point index for compact maps defined on cones in a Banach space $X$ (see [23]).

Lemma 2.4. Assume that $A: \bar{P}_{\rho} \rightarrow P$ is a compact map. Then the following results hold:

1) If there exists $x_{0} \in P \backslash\{0\}$ such that $u \neq A u+v x_{0}$ for $u \in \partial P_{\rho}$ and $v \geq 0$, then $i_{\rho}\left(A, P_{\rho}\right)=0$.

2) If $u \neq \zeta A u$ for $u \in \partial P_{\rho}$ and $\zeta \in(0,1]$, then $i_{\rho}\left(A, P_{\rho}\right)=1$.

$3)$ If $i_{\rho}\left(A, P_{\rho}\right)=1$ and $i_{\rho}\left(A, P_{\rho_{0}}\right)=0$ for some $\rho_{0} \in(0, \rho)$, then $A$ has a fixed point in $P_{\rho} \backslash \bar{P}_{\rho_{0}}$.

Now, we are in a position to give our main result and proof analogous results were established in [24].

Theorem 2.1. Assume that $(H 1)$ - $(H 5)$ holds. $\mu_{1} \in(0, \infty)$ be the same as in (2.5). Assume that the following conditions hold:

$\left(\left(\mathrm{f}_{i_{0}}\right)_{0}\right)_{\rho_{0}}$. There exist $i_{0} \in(1, \cdots, n), \quad \varepsilon>0$ and $\rho_{0}>0$ such that $f_{i_{0}}(t, u) \geq\left(\mu_{1}+\varepsilon\right) u_{i_{0}}$ for $t \in[0, T]$ and all $u \in \mathbb{R}_{+}^{n}$ with $|u| \in\left[0, \rho_{0}\right]$.

$\left(\left(\mathrm{f}_{\mathrm{i}}^{\infty}\right)\right)_{\rho_{1}}$. There exist $\varepsilon>0$ and $\rho_{1}>0$ such that for $i \in(1, \cdots, n), \quad f_{i}(t, u) \leq\left(\mu_{1}-\varepsilon\right)|u|$ for $t \in[0, T]$ and all $u \in \mathbb{R}_{+}^{n}$ with $|u| \geq \rho_{1}$. Then (1.1) has a nonzero positive solution in $P$.

Proof. By Lemma 2.1, Lemma 2.2 and Lemma 2.3, $L: C\left([0, T] ; \mathbb{R}^{n}\right) \rightarrow C\left([0, T] ; \mathbb{R}^{n}\right)$ is compact and satisfies $L(P) \subset P$.

This, together with the continuity of $f_{i}$ in (H3), implies that $A: P \rightarrow P$ is compact. Without loss of generalization, we assume that $u \neq A u$ for $u \in \partial P_{\rho_{0}}$. Let $\varphi=\left(\varphi_{1}, \cdots, \varphi_{1}\right)$, where $\varphi_{1}$ is the same as in (2.5). We prove that

$$
u \neq A u+v \varphi, \forall u \in \partial P_{\rho_{0}} v \geq 0 .
$$

In fact, if not, there exist $u \in \partial P_{\rho_{0}}$ and $v \geq 0$ such that $u=A u+v \varphi$. Then

$$
u_{i_{0}}(t)=\Gamma\left(f_{i_{0}}(t, u(t))\right)+v \varphi_{1}, \forall t \in[0, T]
$$

It follows that $u_{i_{0}}(t) \geq v \varphi_{1}$ for $\forall t \in[0, T]$. Let

$$
\tau=\sup \left\{\tau_{0} \geq 0: u_{i_{0}}(t) \geq \tau_{0} \varphi_{1}, \forall t \in[0, T]\right\} .
$$

Then $0<v \leq \tau<\infty \quad$ and $\quad u_{i_{0}}(t) \geq \tau \varphi_{1}, \quad \forall t \in[0, T]$. This, together with (2.12), $\left(\left(\mathrm{f}_{i_{0}}\right)_{0}\right)_{\rho_{0}}$ and (2.5), implies that for all $t \in[0, T]$

$$
\begin{aligned}
u_{i_{0}}(t) & =\Gamma\left(\left(\mu_{1}+\varepsilon\right) u_{i_{0}}(t)\right) \geq\left(\mu_{1}+\varepsilon\right) \tau \Gamma \varphi_{1}(t) \\
& \geq\left(\mu_{1}+\varepsilon\right) \tau\left(\varphi_{1}(t) / \mu_{1}\right)
\end{aligned}
$$

Hence, we have $\tau \geq\left(\mu_{1}+\varepsilon\right)\left(\tau / \mu_{1}\right)$, a contradiction. It follows from (2.11) and Lemma 2.4 (1) $i_{\rho}\left(A, P_{\rho}\right)=0$.

For each $i \in(1, \cdots, n)$, by the continuity of $f_{i}$, there exists $b_{i}>0$ such that $f_{i}(t, u) \leq b_{i}$ for $t \in[0, T]$, $u \in \mathbb{R}_{+}^{n}$ with $|u| \leq \rho_{1}$.

This, together with $\left(\left(\mathrm{f}_{\mathrm{i}}^{\infty}\right)\right)_{\rho_{1}}$ implies that, for each $i \in(1, \cdots, n) \quad f_{i}(t, u) \leq b_{i}+\left(\mu_{1}-\varepsilon\right)|u|$ for $t \in[0, T]$ and all

$$
u \in \mathbb{R}_{+}^{n}
$$

Since

$$
\left(r\left(\mu_{1}-\varepsilon\right) \Gamma\right)=\left(\mu_{1}-\varepsilon\right) r(\Gamma)<1,
$$

$\left(I-\left(\mu_{1}-\varepsilon\right) \Gamma\right)^{-1}$ exists and is bounded and satisfies $\left(I-\left(\mu_{1}-\varepsilon\right) \Gamma\right)^{-1} P_{1} \subset P_{1}$.

Let $\hat{b}_{i}=b_{i}$ for $t \in[0, T]$,

$$
\rho_{1}=\max \left\{\left\|\Gamma \hat{b}_{i}\right\|: i \in(1, \cdots, n)\right\}
$$

and

$$
\rho^{*}=\left\|\left(I-\left(\mu_{1}-\varepsilon\right) \Gamma\right)^{-1} \hat{\rho}_{1}\right\|,
$$

where $\hat{\rho}_{1}(t)=\rho_{1}$ for $t \in[0, T]$. Let $\rho>\rho^{*}$. We prove

$$
u \neq \zeta A u, u \in \partial P_{\rho}, \zeta \in(0,1] .
$$

Indeed, if not, there exist $u \in \partial P_{\rho}$ and $\zeta \in(0,1]$ such that $u \neq \zeta A u$. By (2.13), we have for each $i \in(1, \cdots, n)$,

$$
u_{i}(t) \leq \rho_{1}+\left(\mu_{1}-\varepsilon\right)(\Gamma|u|)(t) \text { for } t \in[0, T],
$$

where $|u|(t)=\max \left\{\left|u_{i}(t)\right|: i \in(1, \cdots, n)\right\}$. Taking the maximum in the above inequality implies that

$$
|u|(t) \leq \rho_{1}+\left(\mu_{1}-\varepsilon\right)(\Gamma|u|)(t)
$$

for $t \in[0, T]$, and

$$
\left(I-\left(\mu_{1}-\varepsilon\right) \Gamma\right)|u|(t) \leq \rho_{1}
$$

for $t \in[0, T]$.

Since

$$
\begin{aligned}
& \left(I-\left(\mu_{1}-\varepsilon\right) \Gamma\right)^{-1} P_{1} \subset P_{1} \\
& |u|(t) \leq\left(I-\left(\mu_{1}-\varepsilon\right) \Gamma\right)^{-1} \hat{\rho}_{1}(t)
\end{aligned}
$$

for $t \in[0, T]$.

Hence, we have

$$
\rho=\|u\|=\max \{|u|(t): t \in[0, T]\} \leq \rho^{*}<\rho .
$$

a contradiction. By (2.14) and Lemma 2.3 (2), 
$i_{\rho}\left(A, P_{\rho}\right)=1$. By Lemma 2.4 (3), (1.1) has a solution in $P_{\rho} \backslash P_{\rho_{0}}$.

\section{Application}

Let the systems

$\left\{\begin{array}{l}u^{\prime \prime}+k^{2} u=\left(a_{i} u_{i}^{1 / 3}+b_{i} u_{i}^{1 / 3}\right) h_{i}\left(\hat{u}_{i}(t)\right), t \in(0, T), T>0, \\ u(0)=\alpha u(T), \\ u^{\prime}(0)=\beta u^{\prime}(T),\end{array}\right.$

where $k \in\left(0, \frac{\pi}{T}\right), \hat{u}_{i}=\left(u_{1}, \cdots, u_{i-1}, u_{i+1}, \cdots, u_{n}\right)$ and

$i \in(1, \cdots, n)$. Assume that the following conditions hold:

1) For each $i \in(1, \cdots, n), a_{i}, b_{i} \geq 0$ and

$h_{i}: \mathbb{R}_{+}^{n-1} \rightarrow \mathbb{R}_{+}^{n}$ is continuous and let

$$
\omega:=\sup \left\{h_{i}\left(\hat{u}_{i}(t)\right): \hat{u}_{i}(t) \in \mathbb{R}_{+}^{n-1}, i \in(1, \cdots, n)\right\}<\infty .
$$

2) There exists $i_{0} \in(1, \cdots, n)$ such that $a_{i_{0}}+b_{i_{0}}>0$ and $\xi:=\min \left\{h_{i}\left(\hat{u}_{i}(t)\right): \hat{u}_{i}(t) \in \mathbb{R}_{+}^{n-1}, i \in(1, \cdots, n)\right\}>0$.

Then equation (3.1) have a nonzero positive solution in $P$.

Proof. For each $i \in(1, \cdots, n)$, we define a function $f_{i}(t, u):[0, T] \times \mathbb{R}_{+}^{n} \rightarrow \mathbb{R}_{+}$by

$$
f_{i}(t, u)=\left(a_{i} u_{i}^{1 / 3}+b_{i} u_{i}^{1 / 3}\right) h_{i}\left(\hat{u}_{i}(t)\right) .
$$

Let $\varepsilon>0$ and

$$
0<\rho_{0} \leq \min \left\{1,\left(\frac{\left(a_{i 0}+b_{i 0}\right) \xi}{\mu_{1}+\varepsilon}\right)^{3 \backslash 2}\right\} .
$$

Then for $t \in[0, T]$ and $u \in \mathbb{R}_{+}^{n}$ with $|u| \in\left[0, \rho_{0}\right]$ and $u_{i} \neq 0$,

$$
\begin{aligned}
f_{i_{0}}(t, u) & \geq\left(\frac{a_{i_{0}}}{u_{i_{0}}^{3 \backslash 2}}+\frac{b_{i_{0}}}{u_{i_{0}}^{3 \backslash 2}}\right) \xi u_{i_{0}} \geq\left(\frac{a_{i_{0}}}{\rho_{0}^{3 \backslash 2}}+\frac{b_{i_{0}}}{\rho_{0}^{3 \backslash 2}}\right) \xi u_{i_{0}} \\
& \geq\left(\mu_{1}+\varepsilon\right) u_{i_{0}} .
\end{aligned}
$$

Hence, $\left(\left(f_{i_{0}}\right)_{0}\right)_{\rho_{0}}$ holds. Let $\rho_{1}>\rho_{0}$,

$$
\rho_{1} \geq \max \left\{\left(\frac{\left(a_{i 0}+b_{i 0}\right) \xi}{\rho}\right)^{3 \backslash 2}, i \in(1, \cdots, n)\right\} \text {. }
$$

Then for $t \in[0, T]$ and $u \in \mathbb{R}_{+}^{n}$ with $|u| \in\left[\rho_{1}, \infty\right]$,

$$
f_{i}(t, u) \leq\left[a_{i}\left|u_{i}^{1 / 3}\right|+b_{i}\left|u_{i}^{1 / 3}\right|\right] u_{i} \leq a_{i}\left|u_{i}^{1 / 3}\right|+b_{i}\left|u_{i}^{1 / 3}\right| \omega
$$

for $i \in(1, \cdots, n)$ it follows that $\left(f_{i}^{\infty}\right)_{\rho_{1}}$ holds. The result follows from Theorem 2.1.

\section{REFERENCES}

[1] L. H. Erbe and P. K. Palamides, "Boundary Value Problems for Second-Order Differential Systems," Journal of Mathematical Analysis and Applications, Vol. 127, No. 1,
1987 , pp. $80-92$.

doi:10.1016/0022-247X(87)90141-7

[2] J. W. Bebernes and K. Schmitt, "Periodic Boundary Value Problems for Systems of Second Order Differential Equations," Journal of Differential Equations, Vol. 13, No. 1, 1973, pp. 32-47. doi:10.1016/0022-0396(73)90030-2

[3] L. H. Erbe and K. Schmitt, "Boundary Value Problems for Second-Order Differential Systems," In: V. Lakshmikantham, Ed., Nonlinear Analysis and Applications, New York and Basel, 1987, pp. 179-183.

[4] H. Wang, "Periodic Solutions to Non-Autonomous Second-Order Systems," Nonlinear Analysis, Vol. 71, No. 3-4, 2009, pp. 1271-1275. doi:10.1016/j.na.2008.11.079

[5] D. Franco and J. R. L. Webb, "Collisionless Orbits of Singular and Nonsingular Dynamical Systems," Discrete and Continuous Dynamical Systems, Vol. 15, No. 3, 2006, pp. 747-757. doi:10.3934/dcds.2006.15.747

[6] R. P. Agarwal, D. O'Regan and P. J. Y. Wong, "Constant-Sign Solutions of a System of Fredholm Integral Equations," Acta Applicandae Mathematicae, Vol. 80, No. 1, 2004, pp. 57-94. doi:10.1023/B:ACAP.0000013257.42126.ca

[7] D. ORegan and H. Wang, "Positive Periodic Solutions of Systems of Second Order Ordinary Differential Equations," Positivity, Vol. 10, No. 2, 2006, pp. 285-298. doi:10.1007/s11117-005-0021-2

[8] X. Lin, D. Jiang, D. ORegan and R. Agarwal, "Twin Positive Periodic Solutions of Second Order Singular Differential Systems," Topological Methods in Nonlinear Analysis, Vol. 25, 2005, pp. 263-273.

[9] L. H. Erbe and K. Schmitt, "On Solvability of Boundary Value Problems for Systems of Differential Equations," Journal of Applied Mathematics and Physics, Vol. 38, 1987.

[10] H. Wang, "Positive Periodic Solutions of Singular Systems with a Parameter," Journal of Differential Equations, Vol. 249, No. 12, 2010, pp. 2986-3002. doi:10.1016/j.jde.2010.08.027

[11] X. Li and Z. Zhang, "On the Existence of Positive Periodic Solutions of Systems of Second Order Differential Equations," Mathematische Nachrichten, Vol. 284, No. 11-12, 2011, pp. 1472-1482. doi:10.1002/mana.200710145

[12] Z. Cao and D. Jiang, "Periodic Solutions of Second Order Singular Coupled Systems," Nonlinear Analysis, Vol. 71, No. 9, 2009, pp. 3661-3667. doi:10.1016/j.na.2009.02.053

[13] J. R. Graef, L. Kong and H. Wang, "A Periodic Boundary Value Problem with Vanishing Greens Function," Applied Mathematics Letters, Vol. 21, No. 2, 2008, pp. 176180. doi:10.1016/j.aml.2007.02.019

[14] I. Rachunkova, M. Tvrdy and I. Vrkoc, "Existence of Nonnegative and Nonpositive Solutions for Second Order Periodic Boundary Value Problems," Journal of Differential Equations, Vol. 176, No. 2, 2001, pp. 445-469. doi:10.1006/jdeq.2000.3995

[15] P. Torres, "Existence of One-Signed Periodic Solutions of Some Second-Order Differential Equations via a Krasnoselskii's Fixed Point Theorem," Journal of Differential 
Equations, Vol. 190, No. 2, 2003, pp. 643-662. doi:10.1016/S0022-0396(02)00152-3

[16] F. Li and Z. Liang, "Existence of Positive Periodic Solutions to Nonlinear Second Order Differential Equations," Applied Mathematics Letters, Vol. 18, No. 11, 2005, pp. 1256-1264. doi:10.1016/i.aml.2005.02.014

[17] D. Jiang, J. Chu, D. ORegan, R. Agarwal, "Multiple Positive Solutions to Superlinear Periodic Boundary Value Problem, with Repulsive Singular Forces," Journal of Mathematical Analysis and Applications, Vol. 286, No. 2, 2003, pp. 563-576. doi:10.1016/S0022-247X(03)00493-1

[18] X. Li and Z. Zhang, "Periodic Solutions for Second-Order Differential Equations with a Singular Nonlinearity," Nonlinear Analysis, Vol. 69, No. 11, 2008, pp. 3866-3876. doi:10.1016/j.na.2007.10.023

[19] D. Jiang, J. Chu and M. Zhang, "Multiplicity of Positive Periodic Solutions to Superlinear Repulsive Singular Equations," Journal of Differential Equations, Vol. 211, No. 2, 2005, pp. 282-302. doi:10.1016/j.jde.2004.10.031

[20] P. J. Torres and M. Zhang, "A Monotone Iterative Scheme for a Nonlinear Second Order Equation Based on a Geeralized Anti-Maximum Principle," Mathematische Nachrichten, Vol. 251, No. 1, 2003, pp. 101-107. doi:10.1002/mana.200310033

[21] R. Ma, "Nonlinear Periodic Boundary Value Problems with Sign-Changing Green's Function," Nonlinear Analysis, Vol. 74, No. 5, 2011, pp. 1714-1720. doi:10.1016/j.na.2010.10.043

[22] B. Liu, L. Liu and Y. Wu, "Existence of Nontrivial Periodic Solutions for a Nonlinear Second Order Periodic Boundary Value Problem," Nonlinear Analysis, Vol. 72, No. 7-8, 2010, pp. 3337-3345. doi:10.1016/j.na.2009.12.014

[23] H. Amann, "Fixed Point Equations and Nonlinear Eigenvalue Problems in Ordered Banach Spaces," SIAM Review, Vol. 18, No. 4, 1976, pp. 620-709.

[24] K. Q. Lan, "Nonzero Positive Solutions of Systems of Elliptic Boundary Value Problems," AMS, Vol. 139, No. 12, 2011, pp. 4343-4349. doi:10.1090/S0002-9939-2011-10840-2

[25] D. D. Hai and H. Wang, "Nontrivial Solutions for p-Laplacian Systems," Journal of Mathematical Analysis and Applications, Vol. 330, No. 1, 2007, pp. 186-194. doi:10.1016/j.jmaa.2006.07.072

[26] K. Q. Lan and W. Lin, "Multiple Positive Solutions of Systems of Hammerstein Integral Equations with Applications to Fractional Differential Equations," Journal London Mathematical Society, Vol. 83 No. 2, 2011, pp. 449-469. doi:10.1112/jlms/jdq090

[27] R. D. Nussbaum, "Eigenvectors of Nonlinear Positive Operators and the Linear Krein-Rutman Theorem, in Fixed Point Theory," In: E. Fadell and G. Fournier, Eds., Lecture Notes in Math, Vol. 886, Springer, 1981, pp. 309-330. 